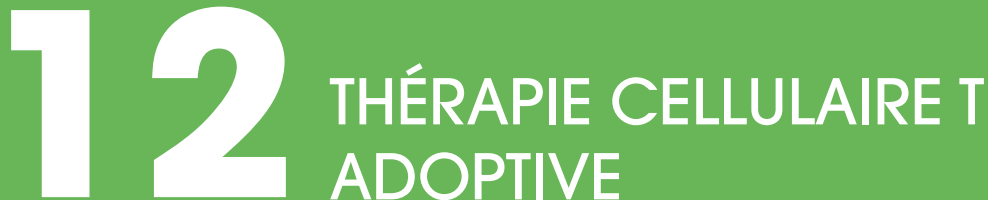

\section{N. Labarriere}

UMR INSERM U892, CRCNA, Nantes, France.

Le transfert adoptif de lymphocytes $T$ spécifiques de tumeurs est une stratégie prometteuse pour le traitement des cancers, et a été utilisée avec succès pour traiter des patients atteints de mélanome métastatique. L'efficacité de ce type de thérapie repose en grande partie sur la spécificité et les propriétés fonctionnelles des lymphocytes T injectés. En effet, les cellules idéales pour une utilisation en thérapie adoptive doivent combiner à la fois des propriétés effectrices antitumorales optimales ainsi que des capacités de persistance et d'amplification in vivo. Depuis plusieurs décennies, les modes de production de ces lymphocytes ont évolué de la simple amplification de lymphocytes infiltrant les tumeurs (TIL) à la production de lymphocytes T spécifiques par tri immunomagnétique ou manipulation génétique. La multiplication attendue des essais de transfert adoptif et leur évaluation clinique et immunologique extensive devrait permettre de définir les caractéristiques optimales des lymphocytes Tà utiliser en termes de spécificité et de fonction, et ainsi d'ouvrir ce type de traitements à de nombreuses tumeurs solides.

\section{SOMMAIRE}

Introduction

1. Transfert adoptif de TIL (lymphocytes infiltrant les tumeurs)

2. Transfert adoptif de lymphocytes $T$ antitumoraux obtenus à partir du sang périphérique

Conclusion

Références bibliographiques 


\section{INTRODUCTION}

Le transfert adoptif de lymphocytes $\mathrm{TCD} 8^{+}$et $\mathrm{CD} 4^{+}$spécifiques d'antigènes représente une stratégie prometteuse pour le traitement des affections virales et des cancers. À l'appui de l'utilisation des lymphocytes T en immunothérapie antitumorale, il existe une corrélation entre la présence d'un infiltrat $T$ au sein de tumeurs solides de diverses origines, telles que les tumeurs ovariennes [1], les cancers colorectaux [2], les cancers du sein [3] et les mélanomes $[4,5]$ et un pronostic favorable. D'autre part, la spécificité de ces lymphocytes T infiltrant les tumeurs (TIL) a été démontrée par l'identification d'antigènes tumoraux reconnus par ces TILs. Cependant, il a été également montré que ces lymphocytes intra-tumoraux ne sont pas fonctionnels in situ, expliquant ainsi leur échec à contrecarrer la progression tumorale [6]. Ce dysfonctionnement peut être induit, d'une part par le microenvironnement tumoral, et notamment par l'absence de signaux de danger [7, 8], et d'autre part par la présence de lymphocytes $T$ régulateurs $C D 4^{+} \mathrm{CD} 25^{+}$qui empêchent la mise en place des réponses immunes effectrices [9]. Le transfert de grandes quantités de lymphocytes T sélectionnés in vitro et fonctionnellement actifs peut permettre de contourner ces mécanismes de tolérance locale et même d'induire in vivo l'amplification de nouveaux lymphocytes T effecteurs antitumoraux $[10,11]$. Cette revue fera le point sur les différentes modalités de transfert adoptif en prenant comme modèle le mélanome métastatique, ainsi que sur les différentes méthodes à l'œuvre pour amplifier des cellules effectrices aux propriétés optimales.

\section{TRANSFERT ADOPTIF DE TIL (LYMPHOCYTES INFILTRANT LES TUMEURS)}

L'administration de TIL amplifiés in vitro a été initiée par l'équipe de S. Rosenberg, à la fin des années 1980. Le premier essai a consisté en l'injection de lymphocytes dérivés de tumeurs de mélanome, avec de fortes doses d'IL-2, à des patients porteurs de mélanome métastatique. Un taux de réponses cliniques d'environ $30 \%$ a été rapporté pour cet essai [12]. Malheureusement, une telle efficacité thérapeutique de l'injection de TIL n'a pas être confirmée ultérieurement dans des essais similaires [13, 14], ce qui soulève entre autres questions celle de l'hétérogénéité des populations de TIL injectées, en termes de statut d'activation et de spécificité. En situation adjuvante, l'injection de TIL spécifiques du mélanome autologue est bénéfique sur la survie sans rechute d'un sous-groupe de patients, porteurs d'un seul ganglion envahi avant l'exérèse chirurgicale [15-17]. Ce bénéfice thérapeutique a été rétrospectivement associé à la présence, parmi les TIL injectés, de lymphocytes T CD8 ${ }^{+}$spécifiques de deux antigènes de mélanome : l'antigène Melan-A/MART-1 [18] et l'antigène MELOE-1 [19]. Ces résultats suggèrent l'efficacité du transfert adoptif de lymphocytes $T$ spécifiques de mélanome en prévention de la rechute pour les patients de stade III. En situation curative, des améliorations décisives de ces traitements ont été apportées dans les années 2000, par l'équipe de S. Rosenberg, d'une part avec l'introduction de traitements lymphodéplétants (chimiothérapie associée ou non à une radiothérapie), préalables au transfert adoptif de TIL, et d'autre part avec une étape de sélection des cultures de TIL

( 210 
réactives à la tumeur [20-22] (figure 1a). Parmi les patients métastatiques réfractaires aux traitements conventionnels ainsi traités, la moitié a montré des réponses objectives avec quelques réponses complètes. Ces réponses sont durables et observées dans tous les organes touchés, même le cerveau. Deux éléments majeurs ont été associés à ces réponses thérapeutiques : la persistance in vivo des cellules T CD8+ injectées [23], et la longueur des télomères des cellules injectées, représentative du nombre de divisions effectuées par ces cellules et conditionnant leur persistance et leur capacité de prolifération in vivo [24]. Dans ces conditions, 6 mois après le transfert adoptif, les lymphocytes T injectés peuvent représenter jusqu'à $75 \%$ des lymphocytes T CD8 ${ }^{+}$ du patient, ce qui montre qu'un environnement lymphopénique favorise la survie et l'expansion de ces lymphocytes, en éliminant d'une part les cellules T régulatrices et en augmentant la disponibilité de cytokines indispensables pour la survie des cellules T, telles que l'IL-15 [25-27].

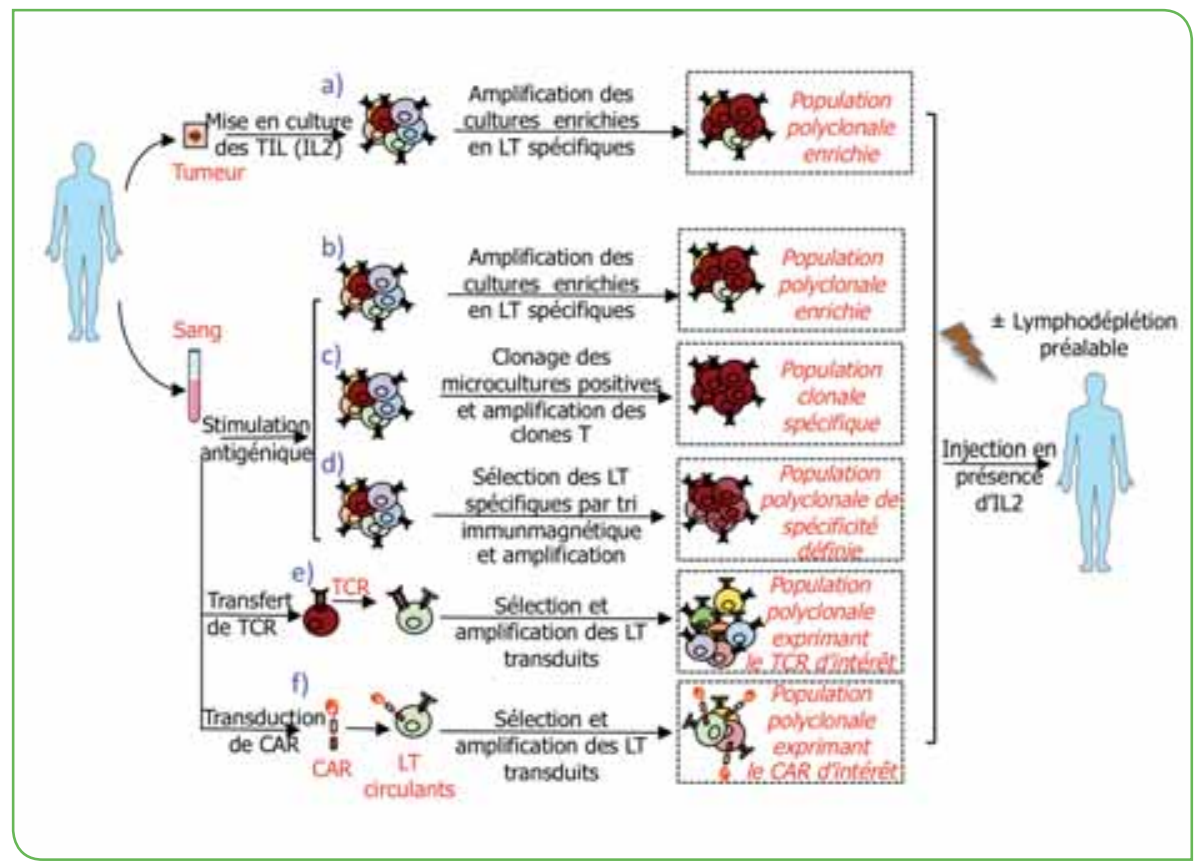

Figure 1 : Modes de production des lymphocytes T spécifiques de tumeurs utilisables dans les essais de transfert adoptif.

a) Les TIL sont produits et amplifiés à partir de fragments de la tumeur autologue, et injectés après lymphodéplétion. b) Après une étape de stimulation antigénique, les lymphocytes T autologues (enrichis en LT spécifiques) sont amplifiés. c) Des lymphocytes d'une spécificité donnée sont obtenus après clonage et amplification. d) Des lymphocytes T polyclonaux d'une spécificité donnée sont obtenus après sélection immunomagnétique et amplification. e) Obtention de T spécifiques par transfert d'un TCR de haute affinité spécifique d'un complexe HLA-peptide donné, et amplification. f) Introduction de CAR dans des lymphocytes T issus de la périphérie et amplification. 


\section{TRANSFERT ADOPTIF DE LYMPHOCYTES T ANTITUMORAUX OBTENUS À PARTIR DU SANG PÉRIPHÉRIQUE}

La production de lymphocytes effecteurs anti-tumoraux à partir de fragments de tumeurs pose le problème de l'accessibilité du matériel tumoral et de la présence de lymphocytes T spécifiques au sein des TIL, ce qui n'est pas systématique. Des méthodes alternatives permettant la production de grands nombres de lymphocytes T spécifiques de tumeurs se sont donc développées, en prenant comme matériel de départ les lymphocytes T du sang périphérique. Dans le sang périphérique, la fréquence des lymphocytes $T$ spécifiques d'antigènes de tumeurs varie de $10^{-4}$ (pour les précurseurs les plus fréquents) à $10^{-7}$ parmi les lymphocytes T effecteurs $\mathrm{CD}^{+}$. Une fréquence de départ relativement élevée facilitera l'amplification des lymphocytes $\mathrm{T}$ spécifiques, et conditionnera donc le choix de l'antigène ciblé. L'étape d'enrichissement en lymphocytes T spécifiques peut être réalisée par une stimulation antigénique suivie d'étapes de sélection ou de clonage. Par ailleurs, des modifications génétiques des lymphocytes $T$ circulants (introduction de récepteurs chimériques à l'antigène (CAR) ou d'un récepteur T spécifique (TCR)) peuvent également être réalisées, ce qui permet de s'affranchir du problème de la fréquence des précurseurs T spécifiques.

\section{2.1. Transfert adoptif de cellules T enrichies en lymphocytes T spécifiques de tumeur}

Un nombre relativement limité d'essais de transfert adoptif a été réalisé avec des lymphocytes de spécificité définie. La majorité de ces études a été effectuée dans le mélanome et a ciblé des antigènes de différenciation mélanocytaire largement exprimés, tels que Melan-A pour lequel le répertoire T spécifique de son épitope HLA-A2 immunodominant est particulièrement abondant chez les patients (de l'ordre de $10^{-4}$ parmi les T CD8 ${ }^{+}$circulants).

L'équipe d'A. Mackensen a été une des premières à réaliser un essai de transfert adoptif de lymphocytes $T$ issus du sang périphérique, fortement enrichis (en moyenne à $50 \%$ ) en effecteurs T spécifiques de cet épitope, à des patients HLA-A2 porteurs de mélanome [28]. Ce transfert de lymphocytes n'était pas précédé d'un traitement lymphodéplétant, mais était accompagné d'injections sous-cutanées d'IL2 (figure 1b). Cette étude a documenté la survie des cellules injectées jusqu'à 14 jours en périphérie, et leur migration dans les tumeurs $[28,29]$. Sur le plan clinique, deux réponses, dont une complète, ont été rapportées, sur 11 patients évaluables. Plus récemment, 10 patients porteurs de mélanome métastatique ont été traités avec des lymphocytes enrichis en $\mathrm{T} \mathrm{CD}^{+}$et $\mathrm{CD} 8^{+}$spécifiques des cellules tumorales et 5 d'entre eux ont présenté des réponses cliniques objectives, ce qui souligne l'efficacité potentielle de

( 212 ) 
cette approche [30]. Néanmoins, l'inconvénient majeur d'une telle stratégie est la co-injection de cellules activées non caractérisées dont l'activité et l'interaction avec les cellules spécifiques in vivo sont méconnues et difficiles à documenter. Les travaux ultérieurs ont donc visé à améliorer la pureté et la spécificité des cellules injectées.

\section{D2.2. Transfert adoptif de populations clonales spécifiques d'antigènes tumoraux}

Le premier essai utilisant des clones T anti-tumoraux dans un essai de transfert adoptif a été celui de l'équipe de S. Rosenberg, publié en 2001 (figure 1c). Dans cette étude, des clones T CD8 ${ }^{+}$spécifiques d'un épitope de l'antigène de différenciation gp100 ont été injectés à des patients porteurs de mélanome métastatique, après lymphodéplétion. Ces lymphocytes se sont avérés incapables de persister chez les patients, et aucune réponse clinique n'a été observée [31, 32]. Deux éléments pourraient expliquer l'échec de cette approche. La méthode de production de ces clones $T$ (issus de stimulations peptidiques répétées, en présence de fortes doses d'IL-2), conduit probablement à un état de sénescence des lymphocytes qui ne sont plus capables de se diviser in vivo. D'autre part, les patients sélectionnés dans cette étude étaient des patients qui avaient préalablement été inclus dans un essai de vaccination avec le même peptide, et qui n'avaient pas répondu à la vaccination, suggérant que le répertoire T spécifique de cet épitope avait un impact limité sur la croissance tumorale.

Parallèlement, un essai de transfert adoptif (ACT) de clones T CD8 ${ }^{+}$spécifiques des antigènes Melan-A et gp100, sans lymphodéplétion préalable, a été réalisé chez 10 patients porteurs de mélanome métastatique [33]. La persistance de ces clones T spécifiques a été documentée, ainsi que des réponses cliniques chez 8 patients.

Une étude similaire a été réalisée par notre équipe chez 14 patients HLA-A2 porteurs d'un mélanome métastatique auxquels ont été injectés des clones T CD8 ${ }^{+}$spécifiques de l'antigène Melan- $A$, sans lymphodéplétion préalable. Les réponses cliniques observées ont été associées non pas à la persistance des cellules injectées, mais à l'expansion in vivo du répertoire T endogène spécifique de Melan-A, documentée chez tous les patients répondeurs $[10,11]$. Ces résultats suggèrent que les clones T injectés ont initié une réponse antitumorale, qui a provoqué l'activation et l'amplification d'autres lymphocytes T spécifiques de tumeurs, dont ceux spécifiques de l'antigène Melan-A, largement exprimé par les cellules tumorales. Ce phénomène d'extension de la réponse immune a également été observé après vaccination peptidique [34,35] et suite au transfert adoptif de clones $\mathrm{T} \mathrm{CD4}^{+}$spécifiques de l'antigène NY-ESO-1, chez des patients atteints de mélanome métastatique [36]. Ce recrutement de lymphocytes T endogènes, qui semble corrélé dans ces différents essais à l'efficacité thérapeutique, pourrait donc être un facteur prédictif de cette efficacité, et est un point crucial à documenter dans le suivi immunologique des patients. Par ailleurs, il est important de noter que cette extension de la réponse immune ne peut être effective qu'en présence d'un système immunitaire fonctionnel, donc en l'absence de lymphodéplétion préalable. 
Néanmoins, l'inconvénient majeur de l'utilisation de clones T en thérapie adoptive est le risque de sélection de clones s'avérant proches de la sénescence, du fait des multiples étapes de culture nécessaires à leur production, et donc peu réactifs in vivo [37]. D'autres stratégies nécessitant moins d'étapes de culture in vitro se sont développées pour la production de cellules de spécificité définie, à un stade de différenciation permettant de préserver leurs capacités de persistance et de multiplication in vivo.

\section{2.3. Transfert adoptif de lymphocytes T spécifiques de tumeur sélectionnés par tri immunomagnétique}

Une alternative au clonage pour obtenir rapidement des lymphocytes T spécifiques moins différenciés est le tri immunomagnétique. Le principe repose sur l'utilisation d'anticorps spécifiques de marqueurs d'activation ou de multimères HLA-peptide se liant à un TCR donné, couplé à des billes magnétiques (figure $1 \mathrm{~d}$ ).

Les lymphocytes spécifiques peuvent être sélectionnés sur la base d'un marqueur d'activation membranaire surexprimé après activation par un stimulus spécifique, tel que la chaîne alpha du récepteur de l'IL-2 (CD25) ou le 4-1BB (CD137), transitoirement surexprimés sur les lymphocytes $T$ activés. L'efficacité d'une sélection basée sur l'expression du 4-1BB a été montrée pour des lymphocytes T spécifiques d'épitopes viraux [38]. Cette méthode permet l'enrichissement en lymphocytes T réactifs, sans toutefois atteindre une pureté totale, ces molécules étant exprimées à un niveau basal sur les cellules au repos.

Une alternative est de sélectionner les lymphocytes T spécifiques après activation, sur la base de la sécrétion de cytokines. Un système de tri magnétique permettant d'enrichir une population T en lymphocytes sécrétant de l'interféron gamma (IFN- $\gamma$ ) a été développé par la société Miltenyi-Biotech. Cette méthode présente l'avantage de trier à la fois des lymphocytes $\mathrm{T} \mathrm{CD4}^{+}$et $\mathrm{CD} 8^{+}$répondant au stimulus antigénique. Elle a été utilisée dans des phases précliniques de sélection de lymphocytes T anti-viraux [39-41] ainsi que de lymphocytes T spécifiques d'antigènes associés à certaines leucémies myéloïdes ou lymphoïdes [42]. Ce principe n'a pas encore été appliqué aux tumeurs solides, pour lesquelles les précurseurs T spécifiques d'épitopes tumoraux sont moins fréquents et qu'il est plus difficile de sélectionner directement à partir du sang périphérique, sans enrichissement préalable. À l'instar du tri sur la base de molécules d'activation, la pureté en cellules spécifiques est rarement atteinte, même si les taux d'enrichissement sont très satisfaisants.

Une autre possibilité pour obtenir des lymphocytes de spécificité définie, en préservant leur potentiel de division in vivo, est l'utilisation de complexes HLA-peptide permettant de trier les lymphocytes T d'intérêt par leur TCR. Le développement de cette technologie a été une étape décisive dans l'analyse des réponses immunes chez les patients, et a également ouvert de nouvelles possibilités pour les traitements de transfert adoptif dans les maladies infectieuses et les cancers. Les premiers essais de transfert de lymphocytes T triés à l'aide de multimères HLA-peptide ont été réalisés avec succès chez des patients porteurs d'infection à cytomégalovirus (CMV), conduisant à une

214 
diminution de la charge virale chez la majorité des patients [43, 44]. À ce jour cette approche n'a pas été tentée en immunothérapie antitumorale, mais notre équipe a mis au point une procédure, utilisable en clinique, permettant la sélection et l'expansion de lymphocytes T spécifiques d'épitopes viraux et tumoraux qui repose sur l'utilisation de complexes HLA-peptides couplés à des billes magnétiques [45-47]. Cette méthode permet la production rapide de lymphocytes $T$ de spécificité parfaitement définie, réactifs vis-à-vis des cellules de mélanome et dont la longueur des télomères est préservée. Un essai de phase I utilisant des lymphocytes T spécifiques de deux antigènes de mélanome, ainsi sélectionnés, est prévu courant 2014.

\section{2.4. Transfert adoptif de lymphocytes T modifiés génétiquement}

\subsection{Transfert de spécificité}

Une stratégie très séduisante qui permet d'obtenir des lymphocytes T fortement réactifs à la tumeur et capables de proliférer in vivo consiste à faire exprimer aux lymphocytes T du sang périphérique un TCR de forte affinité spécifique d'un antigène de tumeur [48]. La faisabilité de ce transfert de spécificité a été initialement démontrée il y a presque 30 ans dans un modèle murin, à l'aide de vecteur rétroviraux [49]. Depuis lors, plusieurs équipes ont transféré des TCR spécifiques d'épitopes de classe I et II dans des lymphocytes issus du sang périphérique, dans le but d'obtenir des lymphocytes T utilisables en thérapie [50-52] (figure 1e). Plus récemment, un essai clinique a été réalisé chez 17 patients porteurs de mélanome métastatique, après lymphodéplétion, avec des lymphocytes de la périphérie transduits avec un TCR spécifique de l'épitope immunodominant Melan-A27-35 [53]. Malgré la persistance des lymphocytes injectés dans la périphérie jusqu'à deux mois après le traitement, seulement 2/15 réponses cliniques objectives ont été documentées. Ces résultats décevants peuvent s'expliquer par un niveau d'expression trop faible du TCR d'intérêt. En effet, les chaînes alpha et bêta du TCR transduit peuvent également s'apparier avec les chaînes du TCR endogène, générant ainsi des spécificités inconnues et des lymphocytes T potentiellement autoréactifs [54]. Ce problème peut être limité en favorisant l'appariement des chaînes transduites soit en introduisant des résidus cystéines dans leurs domaines constants pour faciliter la formation de ponts disulfure intra-chaînes, soit en fusionnant les chaînes $\alpha \beta$ avec le CD3- $\varepsilon$ [55-57]. Plus récemment, cette approche a montré son efficacité chez un patient atteint de cancer colique chez qui ont été transférés des lymphocytes $T$ transduits avec un récepteur spécifique de l'antigène carcino-embryonnaire (CEA) [58]. Néanmoins, la réponse thérapeutique était associée à une réaction inflammatoire colique sévère liée à l'expression de l'antigène par les cellules de l'épithélium colique, soulignant les risques de cette approche. En effet, de par leur haute affinité, les TCR transgéniques eux-mêmes peuvent être responsables de réactions autoimmunes. C'est également ce qui a été observé dans un essai récent, dans lequel il a été montré que les TCR transduits spécifiques de l'antigène MAGE-A3 pouvaient cross-réagir avec un épitope présentant des similarités avec l'épitope ciblé et exprimé par les cardiomyocytes, provoquant ainsi des accidents létaux [59]. 


\subsubsection{Transfert de CAR}

Une stratégie alternative au transfert de TCR est la transduction des lymphocytes T autologues par des récepteurs chimérique à l'antigène (CAR). Un récepteur chimérique est constitué de la partie variable d'un anticorps spécifique d'un antigène tumoral de surface, couplé à un ou plusieurs domaines de signalisation [60]. Les CAR de première génération sont constitués de cette partie variable liée au domaine de signalisation CD3z. Ceux de deuxième et troisième génération contiennent un ou plusieurs domaines de co-stimulation additionnels (figure 1f). Les lymphocytes T ainsi transduits présentent, outre des propriétés cytotoxiques spécifiques, des capacités accrues de persistance, prolifération et sécrétion de cytokines. L'avantage de cette approche est qu'elle permet de s'affranchir de la restriction HLA des TCR, et donc de cibler une plus large catégorie de patients. Un des premiers essais utilisant des CAR en immunothérapie antitumorale a été réalisé dans le glioblastome, ciblant l'antigène GD2 [61] et a mis en évidence 1 réponse complète sur 11 patients traités. D'autres essais ciblant les antigènes CD19 [62] et CD20 [63] ont été réalisés chez des patients porteurs de lymphomes. Des réponses cliniques y ont été observées, sans toxicité majeure associée. En revanche, le ciblage d'un antigène exprimé à bas bruit par des tissus sains peut conduire à une activation incontrôlée de lymphocytes T transduits par des CAR. C'est le cas de l'antigène Her2-neu, ciblé par des CAR de troisième génération dans le cadre d'un essai réalisé chez un patient atteint de cancer colique. L'injection des lymphocytes T transformés a été très rapidement suivie d'une " tempête cytokinique » ayant entraîné le décès, probablement provoquée par la reconnaissance d'un faible niveau d'antigène exprimé sur les cellules de l'épithélium pulmonaire [64].

Bien qu'extrêmement séduisantes, les approches de transfert de lymphocytes T génétiquement modifiés sont complexes à mettre en œuvre, tant sur le plan de la méthodologie que sur le plan de la sécurité des patients, deux points en cours d'optimisation.

\section{CONCLUSION}

Les nombreuses études d'ACT pour le traitement des cancers ont permis d'en démontrer la faisabilité et de documenter dans certains cas une efficacité thérapeutique. Le transfert adoptif de lymphocytes T spécifiques de tumeurs a donc d'ores et déjà une place parmi l'arsenal des traitements du mélanome métastatique, notamment en situation adjuvante. L'amélioration des résultats thérapeutiques du transfert adoptif en traitement curatif reste nécessaire et viendra probablement de futurs protocoles combinant le transfert adoptif et d'autres approches telles que la vaccination thérapeutique, le blocage de certaines molécules inhibitrices (CTLA-4 ou PD-1) ou encore l'utilisation de drogues épigénétiques permettant une expression accrue des HLA ou des antigènes.

\section{( 216}




\section{Déférences bibliographiques}

1. Sato E., Olson S.H., Ahn J., Bundy B., Nishikawa H., Qian F., et al. (2005) Intraepithelial CD8+ tumor-infiltrating lymphocytes and a high $\mathrm{CD} 8+/$ regulatory $\mathrm{T}$ cell ratio are associated with favorable prognosis in ovarian cancer. Proc. Natl. Acad. Sci. U S A. 102: 18538-43.

2. Galon J., Costes A., Sanchez-Cabo F., Kirilovsky A., Mlecnik B., Lagorce-Pages C., et al. (2006) Type, density, and location of immune cells within human colorectal tumors predict clinical outcome. Science 313: 1960-4.

3. Marrogi A.J., Munshi A., Merogi A.J., Ohadike Y., El-Habashi A., Marrogi O.L., et al. (1997) Study of tumor infiltrating lymphocytes and transforming growth factor-beta as prognostic factors in breast carcinoma. Int. J. Cancer. 74: 492-501.

4. Clark W.H., Jr., Elder D.E., Guerry D.t., Braitman L.E., Trock B.J., Schultz D., et al. (1989) Model predicting survival in stage I melanoma based on tumor progression. J. Natl. Cancer Inst. 81: 1893-904.

5. Clemente C.G., Mihm M.C., Jr., Bufalino R., Zurrida S., Collini P., Cascinelli N. (1996) Prognostic value of tumor infiltrating lymphocytes in the vertical growth phase of primary cutaneous melanoma. Cancer 77: 1303-10.

6. Zippelius A., Batard P., Rubio-Godoy V., Bioley G., Lienard D., Lejeune F, et al. (2004) Effector function of human tumor-specific CD8 T cells in melanoma lesions: a state of local functional tolerance. Cancer Res. 64: 2865-73.

7. Matzinger P. (2002) The danger model: a renewed sense of self. Science 296: 301-5

8. Ochsenbein A.F., Sierro S., Odermatt B., Pericin M., Karrer U., Hermans J., et al. (2001) Roles of tumour localization, second signals and cross priming in cytotoxic T-cell induction. Nature 411: 1058-64.

9. Shevach E.M. (2002) CD4+ CD25+ suppressor T cells: more questions than answers. Nat. Rev. Immunol. 2: 389-400.

10. Khammari A., Labarriere N., Vignard V., Nguyen J.M., Pandolfino M.C., Knol A.C., et al. (2009) Treatment of metastatic melanoma with autologous Melan-A/MART-1-specific cytotoxic T lymphocyte clones. J. Invest. Dermatol. 129: 2835-42.

11. Vignard V., Lemercier B., Lim A., Pandolfino M.C., Guilloux Y., Khammari A., et al. (2005) Adoptive transfer of tumor-reactive Melan-A-specific CTL clones in melanoma patients is followed by increased frequencies of additional Melan-A-specific T cells. J. Immunol. 175: 4797-805.

12. Rosenberg S.A., Packard B.S., Aebersold P.M., Solomon D., Topalian S.L., Toy S.T., et al. (1988) Use of tumor-infiltrating lymphocytes and interleukin-2 in the immunotherapy of patients with metastatic melanoma. A preliminary report. N. Engl. J. Med. 319: 1676-80.

13. Dillman R.O., Oldham R.K., Barth N.M., Cohen R.J., Minor D.R., Birch R., et al. (1991) Continuous interleukin-2 and tumor-infiltrating lymphocytes as treatment of advanced melanoma. A national biotherapy study group trial. Cancer 68: 1-8.

14. Goedegebuure P.S., Douville L.M., Li H., Richmond G.C., Schoof D.D., Scavone M., et al. (1995) Adoptive immunotherapy with tumor-infiltrating lymphocytes and interleukin-2 in patients with metastatic malignant melanoma and renal cell carcinoma: a pilot study. J. Clin. Oncol. 13: 193949.

15. Dreno B., Nguyen J.M., Khammari A., Pandolfino M.C., Tessier M.H., Bercegeay S., et al. (2002) Randomized trial of adoptive transfer of melanoma tumor-infiltrating lymphocytes as adjuvant therapy for stage III melanoma. Cancer Immunol. Immunother. 51: 539-46.

16. Khammari A., Nguyen J.M., Pandolfino M.C., Quereux G., Brocard A., Bercegeay S., et al. (2007) Long-term follow-up of patients treated by adoptive transfer of melanoma tumor-infiltrating lymphocytes as adjuvant therapy for stage III melanoma. Cancer Immunol. Immunother. 56: 185360. 
17. Labarriere N., Pandolfino M.C., Gervois N., Khammari A., Tessier M.H., Dreno B., et al. (2002) Therapeutic efficacy of melanoma-reactive TIL injected in stage III melanoma patients. Cancer Immunol. Immunother. 51: 532-8.

18. Benlalam H., Vignard V., Khammari A., Bonnin A., Godet Y., Pandolfino M.C., et al. (2007) Infusion of Melan-A/Mart-1 specific tumor-infiltrating lymphocytes enhanced relapse-free survival of melanoma patients. Cancer Immunol. Immunother. 56: 515-26.

19. Godet Y., Moreau-Aubry A., Guilloux Y., Vignard V., Khammari A., Dreno B., et al. (2008) MELOE-1 is a new antigen overexpressed in melanomas and involved in adoptive $T$ cell transfer efficiency. J. Exp. Med. 205: 2673-82.

20. Dudley M.E., Wunderlich J.R., Robbins P.F., Yang J.C., Hwu P., Schwartzentruber D.J., et al. (2002) Cancer regression and autoimmunity in patients after clonal repopulation with antitumor lymphocytes. Science 298: 850-4.

21. Dudley M.E., Wunderlich J.R., Yang J.C., Sherry R.M., Topalian S.L., Restifo N.P., et al. (2005) Adoptive cell transfer therapy following non-myeloablative but lymphodepleting chemotherapy for the treatment of patients with refractory metastatic melanoma. J. Clin. Oncol. 23: 2346-57.

22. Rosenberg S.A., Yang J.C., Sherry R.M., Kammula U.S., Hughes M.S., Phan G.Q., et al. (2011) Durable complete responses in heavily pretreated patients with metastatic melanoma using T-cell transfer immunotherapy. Clin. Cancer Res. 17: 4550-7.

23. Robbins P.F., Dudley M.E., Wunderlich J., El-Gamil M., Li Y.F., Zhou J., et al. (2004) Cutting edge: persistence of transferred lymphocyte clonotypes correlates with cancer regression in patients receiving cell transfer therapy. J. Immunol. 173: 7125-30.

24. Zhou J., Shen X., Huang J., Hodes R.J., Rosenberg S.A., Robbins P.F. (2005) Telomere length of transferred lymphocytes correlates with in vivo persistence and tumor regression in melanoma patients receiving cell transfer therapy. J. Immunol. 175: 7046-52.

25. Antony P.A., Piccirillo C.A., Akpinarli A., Finkelstein S.E., Speiss P.J., Surman D.R., et al. (2005) CD8+ $T$ cell immunity against a tumor/self-antigen is augmented by CD4+ T helper cells and hindered by naturally occurring T regulatory cells. J. Immunol. 174: 2591-601.

26. Dudley M.E., Yang J.C., Sherry R., Hughes M.S., Royal R., Kammula U, et al. (2008) Adoptive cell therapy for patients with metastatic melanoma: evaluation of intensive myeloablative chemoradiation preparative regimens. J. Clin. Oncol. 26: 5233-9.

27. Gattinoni L., Finkelstein S.E., Klebanoff C.A., Antony P.A., Palmer D.C., Spiess P.J., et al. (2005) Removal of homeostatic cytokine sinks by lymphodepletion enhances the efficacy of adoptively transferred tumor-specific CD8+ T cells. J. Exp. Med. 202: 907-12.

28. Meidenbauer N., Marienhagen J., Laumer M., Vogl S., Heymann J., Andreesen R., et al. (2003) Survival and tumor localization of adoptively transferred Melan-A-specific T cells in melanoma patients. J. Immunol. 170: 2161-9.

29. Mackensen A., Meidenbauer N., Vogl S., Laumer M., Berger J., Andreesen R. (2006) Phase I study of adoptive T-cell therapy using antigen-specific CD8+ T cells for the treatment of patients with metastatic melanoma. J. Clin. Oncol. 24: 5060-9.

30. Verdegaal E.M., Visser M., Ramwadhdoebe T.H., van der Minne C.E., van Steijn J.A., Kapiteijn E., et al. (2011) Successful treatment of metastatic melanoma by adoptive transfer of blood-derived polyclonal tumor-specific CD4+ and CD8+ T cells in combination with low-dose interferon-alpha. Cancer Immunol. Immunother. 60: 953-63.

31. Dudley M.E., Wunderlich J., Nishimura M.I., Yu D., Yang J.C., Topalian S.L., et al. (2001) Adoptive transfer of cloned melanoma-reactive $\mathrm{T}$ lymphocytes for the treatment of patients with metastatic melanoma. J. Immunother. 24: 363-73.

32. Dudley M.E., Wunderlich J.R., Yang J.C., Hwu P., Schwartzentruber D.J., Topalian S.L., et al. (2002) A phase I study of nonmyeloablative chemotherapy and adoptive transfer of autologous tumor antigen-specific T lymphocytes in patients with metastatic melanoma. J. Immunother. 25: 243-51.

\section{8}


33. Yee C., Thompson J.A., Byrd D., Riddell S.R., Roche P., Celis E., et al. (2002) Adoptive T cell therapy using antigen-specific CD8+ T cell clones for the treatment of patients with metastatic melanoma: in vivo persistence, migration, and antitumor effect of transferred T cells. Proc. Natl. Acad. Sci. U S A 9: 16168-73.

34. Germeau C., Ma W., Schiavetti F., Lurquin C., Henry E., Vigneron N., et al. (2005) High frequency of antitumor T cells in the blood of melanoma patients before and after vaccination with tumor antigens. J. Exp. Med. 201: 241-8.

35. Lurquin C., Lethe B., De Plaen E., Corbiere V., Theate I., van Baren N., et al. (2005) Contrasting frequencies of antitumor and anti-vaccine $T$ cells in metastases of a melanoma patient vaccinated with a MAGE tumor antigen. J. Exp. Med. 201: 249-57.

36. Hunder N.N., Wallen H., Cao J., Hendricks D.W., Reilly J.Z., Rodmyre R., et al. (2008) Treatment of metastatic melanoma with autologous CD4+ T cells against NY-ESO-1. N. Engl. J. Med. 358: 2698-703.

37. Hinrichs C.S., Gattinoni L., Restifo N.P. (2006) Programming CD8+ T cells for effective immunotherapy. Curr. Opin. Immunol. 18: 363-70.

38. Wehler T.C., Karg M., Distler E., Konur A., Nonn M., Meyer R.G., et al. (2008) Rapid identification and sorting of viable virus-reactive $\mathrm{CD} 4(+)$ and $\mathrm{CD} 8(+) \mathrm{T}$ cells based on antigen-triggered CD137 expression. J. Immunol. Methods 339: 23-37.

39. Feuchtinger T., Lang P., Hamprecht K., Schumm M., Greil J., Jahn G., et al. (2004) Isolation and expansion of human adenovirus-specific CD4+ and CD8+ T cells according to IFN-gamma secretion for adjuvant immunotherapy. Exp. Hematol. 32: 282-9.

40. Feuchtinger T., Richard C., Joachim S., Scheible M.H., Schumm M., Hamprecht K., et al. (2008) Clinical grade generation of hexon-specific $T$ cells for adoptive T-cell transfer as a treatment of adenovirus infection after allogeneic stem cell transplantation. J. Immunother. 31: 199-206.

41. Rauser G., Einsele H., Sinzger C., Wernet D., Kuntz G., Assenmacher M., et al. (2004) Rapid generation of combined CMV-specific CD4+ and CD8+ T-cell lines for adoptive transfer into recipients of allogeneic stem cell transplants. Blood 103: 3565-72.

42. Jedema I., Meij P., Steeneveld E., Hoogendoorn M., Nijmeijer B.A., van de Meent M., et al. (2007) Early detection and rapid isolation of leukemia-reactive donor T cells for adoptive transfer using the IFN-gamma secretion assay. Clin. Cancer Res. 13: 636-43.

43. Cobbold M., Khan N., Pourgheysari B., Tauro S., McDonald D., Osman H., et al. (2005) Adoptive transfer of cytomegalovirus-specific CTL to stem cell transplant patients after selection by HLApeptide tetramers. J. Exp. Med. 202: 379-86.

44. Schmitt A., Tonn T., Busch D.H., Grigoleit G.U., Einsele H., Odendahl M., et al. (2011) Adoptive transfer and selective reconstitution of streptamer-selected cytomegalovirus-specific CD8+ T cells leads to virus clearance in patients after allogeneic peripheral blood stem cell transplantation. Transfusion 51: 591-9.

45. Bodinier M., Peyrat M.A., Tournay C., Davodeau F., Romagne F., Bonneville M., et al. (2000) Efficient detection and immunomagnetic sorting of specific T cells using multimers of MHC class I and peptide with reduced CD8 binding. Nat. Med. 6: 707-10.

46. Bouquie R., Bonnin A., Bernardeau K., Khammari A., Dreno B., Jotereau F., et al. (2009) A fast and efficient HLA multimer-based sorting procedure that induces little apoptosis to isolate clinical grade human tumor specific T lymphocytes. Cancer Immunol. Immunother. 58: 553-66.

47. Labarriere N., Gervois N., Bonnin A., Bouquie R., Jotereau F., Lang F. (2008) PBMC are as good a source of tumor-reactive T lymphocytes as TIL after selection by Melan-A/A2 multimer immunomagnetic sorting. Cancer Immunol. Immunother. 57: 185-95.

48. Schumacher T.N. (2002) T-cell-receptor gene therapy. Nat. Rev. Immunol. 2:512-9.

49. Dembic Z., Haas W., Weiss S., McCubrey J., Kiefer H., von Boehmer H., et al. (1986) Transfer of specificity by murine alpha and beta T-cell receptor genes. Nature 320: 232-8. 
50. Calogero A., de Leij L.F., Mulder N.H., Hospers G.A. (2000) Recombinant T-cell receptors: an immunologic link to cancer therapy. J. Immunother. 23: 393-400.

51. Fujio K., Misaki Y., Setoguchi K., Morita S., Kawahata K., Kato I., et al. (2000) Functional reconstitution of class II MHC-restricted T cell immunity mediated by retroviral transfer of the alpha beta TCR complex. J. Immunol. 165: 528-32.

52. Stanislawski T., Voss R.H., Lotz C., Sadovnikova E., Willemsen R.A., Kuball J., et al. (2001) Circumventing tolerance to a human MDM2-derived tumor antigen by TCR gene transfer. Nat. Immunol. 2: 962-70.

53. Morgan R.A., Dudley M.E., Wunderlich J.R., Hughes M.S., Yang J.C., Sherry R.M., et al. (2006) Cancer regression in patients after transfer of genetically engineered lymphocytes. Science 314: 126-9.

54. Bendle G.M., Linnemann C., Hooijkaas A.I., Bies L., de Witte M.A., Jorritsma A., et al. (2010) Lethal graft-versus-host disease in mouse models of T cell receptor gene therapy. Nat. Med. 16: 565-70, $1 p$ following 70

55. Cohen C.J., Li Y.F., El-Gamil M., Robbins P.F., Rosenberg S.A., Morgan R.A. (2007) Enhanced antitumor activity of $T$ cells engineered to express T-cell receptors with a second disulfide bond. Cancer Res. 67: 3898-903.

56. Cohen C.J., Zhao Y., Zheng Z., Rosenberg S.A., Morgan R.A. (2006) Enhanced antitumor activity of murine-human hybrid T-cell receptor (TCR) in human lymphocytes is associated with improved pairing and TCR/CD3 stability. Cancer Res. 66: 8878-86.

57. Kuball J., Dossett M.L., Wolfl M., Ho W.Y., Voss R.H., Fowler C., et al. (2007) Facilitating matched pairing and expression of TCR chains introduced into human T cells. Blood 109: 2331-8.

58. Parkhurst M.R., Yang J.C., Langan R.C., Dudley M.E., Nathan D.A., Feldman S.A., et al. (2011) $T$ cells targeting carcinoembryonic antigen can mediate regression of metastatic colorectal cancer but induce severe transient colitis. Mol. Ther. 19: 620-6.

59. Linette G.P., Stadtmauer E.A., Maus M.V., Rapoport A.P., Levine B.L., Emery L., et al. (2013) Cardiovascular toxicity and titin cross-reactivity of affinity-enhanced $\mathrm{T}$ cells in myeloma and melanoma. Blood 22: 863-71.

60. Lipowska-Bhalla G., Gilham D.E., Hawkins R.E., Rothwell D.G. (2012) Targeted immunotherapy of cancer with CAR T cells: achievements and challenges. Cancer Immunol. Immunother. 61: 95362.

61. Pule M.A., Savoldo B., Myers G.D., Rossig C., Russell H.V., Dotti G., et al. (2008) Virus-specific $T$ cells engineered to coexpress tumor-specific receptors: persistence and antitumor activity in individuals with neuroblastoma. Nat. Med. 14: 1264-70.

62. Kochenderfer J.N., Wilson W.H., Janik J.E., Dudley M.E., Stetler-Stevenson M., Feldman S.A., et al. (2010) Eradication of B-lineage cells and regression of lymphoma in a patient treated with autologous T cells genetically engineered to recognize CD19. Blood 16: 4099-102.

63. Till B.G., Jensen M.C., Wang J., Chen E.Y., Wood B.L., Greisman H.A., et al. (2008) Adoptive immunotherapy for indolent non-Hodgkin lymphoma and mantle cell lymphoma using genetically modified autologous CD20-specific T cells. Blood 112: 2261-71.

64. Morgan R.A., Yang J.C., Kitano M., Dudley M.E., Laurencot C.M., Rosenberg S.A. (2010) Case report of a serious adverse event following the administration of $T$ cells transduced with a chimeric antigen receptor recognizing ERBB2. Mol. Ther. 18: 843-51. 